\title{
Wake-sleep, thermoregulatory, and autonomic effects of cholinergic activation of the lateral hypothalamus in the rat: a pilot study
}

\author{
A. DI CRISTOFORO, M. CERRI, F. DEL VECCHIO, T. HITREC, M. LUPPI, E. PEREZ, \\ G. ZAMBONI, R. AMICI
}

Dept. Biomedical and NeuroMotor Sciences - Physiology, Alma Mater Studiorum, University of Bologna, Italy

\author{
A B S T R A C T
}

A major role in the wake-promoting effects of the activation of the lateral hypothalamus (LH) has been ascribed to a population of orexin (ORX)-containing neurons that send projections to central areas which regulate Wake-Sleep and autonomic function. Since, in the rat, a substantial amount of ORX neurons receive cholinergic projections from cells involved in Wake-Sleep regulation, the aim of this study was to assess the role played by LH cholinoceptive cells in Wake-Sleep and autonomic regulations. To this end, the effects of a microinjection of the cholinergic agonist Carbachol (CBL) into the LH were compared to those obtained through the activation of a wider cell population by the microinjection of the GABA antagonist GABAzine (GBZ). The results of this pilot study showed that both drugs elicited the same behavioral and autonomic effects, those caused by GBZ being larger and longer-lasting than those following administration of CBL. Briefly, wakefulness was enhanced and sleep was depressed, and brain temperature and heart rate consistently increased, while mean arterial pressure showed only a mild increment. Surprisingly, the administration of the drug vehicle (SAL) elicited a similar pattern of Wake-Sleep effects which, although much smaller, were sufficient to mask any statistical significance between treatment and control data. In conclusion, the results of this work show that the arousal elicited by LH disinhibition by GABAzine is concomitant with autonomic responses set by the intervention of cold-defense mechanisms. Since the same response is elicited at a lower level by CBL administration, the hypothesis of an involvement of cholinoceptive ORX neurons in its generation is discussed.

Key words

Lateral hypothalamus $\bullet$ Cholinergic system $\bullet$ Wake-Sleep states $\bullet$ Thermoregulation $\bullet$ Autonomic function

\section{Introduction}

The lateral hypothalamus (LH) contains a heterogeneous neuronal population that is involved in behavioral state control, the maintenance of energy homeostasis, and the regulation of the autonomic outflow to several target organs (Berthoud and Munzberg, 2011). In the rat, the overall activation of LH neurons has been shown to promote arousal and the maintenance of the waking state (Berthoud and Munzberg, 2011), to favor specific waking behaviors, such as locomotion, feeding and drinking (Li et al., 2011), and to activate the sympathetic outflow to the brown adipose tissue (BAT) and to the heart (Cerri and Morrison, 2005). As far as the whole set of behavioral states is concerned, the pharmacological inhibition of LH in the rat has recently been shown to promote the occurrence of a sustained bout of non-rapid-eye movement (NREM) sleep (Clement et al., 2012) with enhanced slow-wave electroencephalographic (EEG) activity (Cerri et al., 2014), suppress REM sleep (Clement et al., 2012; Cerri et al., 2014), and lead to a transient inhibition of autonomic cold-defense mechanisms (Cerri et al, 2014). 
In the regulation of the physiological functions and behavioral state control carried out by LH neurons, a central role is known to be played by a cluster of orexin (ORX)-containing neurons, that send widespread projections to several brain areas that are involved in the control of behavioral arousal and autonomic function (Yoshida et al., 2006, Alexandre et al., 2013); furthermore, ORX has been shown to affect cardiovascular functions (Shirasaka et al., 2002) and to regulate thermoregulatory sympathetic outflow (Tupone et al., 2011).

The coordination of behavioral arousal with an adequate autonomic activation by ORX neurons requires a set of reciprocal connections with other brain areas. Among these, the reciprocal connections of ORX neurons with cholinergic neurons in the Basal Forebrain (BF) emerge as being of importance, since they may constitute a positive feedback circuit contributing to the consolidation of behavioral states (Sakurai et al., 2005). Although the cholinergic input to ORX neurons also appears to arise outside BF (Henny and Jones, 2006), cholinergic neurons of BF have been shown to both promote cortical activation during active wakefulness and REM sleep (Lee et al., 2005) and to be strongly excited by ORX neurons (Eggerman et al., 2001). It has also been observed that a local delivery of the cholinomimetic drug Carbachol (CBL) depolarizes the majority of ORX neurons in vitro (Sakurai et al., 2005).

Since no report exists concerning the effects of the activation of cholinergic receptors of LH neurons in the free-behaving rat, the present pilot study was aimed at evaluating the behavioral and autonomic effects evoked by a selective pharmacological stimulation of the LH cholinoceptive neurons through the local administration of CBL. To evaluate the relative power of this activation, the behavioral and autonomic effects induced by CBL were compared with those evoked by the activation of a larger population of $\mathrm{LH}$ neurons, obtained through the local administration of the $\mathrm{GABA}_{\mathrm{A}}$ antagonist GABAzine (GBZ).

\section{Methods}

\section{Animals}

Male CD Sprague-Dawley rats $(n=8$, weight 250$300 \mathrm{~g}$ ) were obtained from Charles River, Inc. (Lecco, Italy), and were kept in normal laboratory conditions (nlab) under a 12h:12h Light-Dark (LD) cycle (Light on at $09.00 \mathrm{~h}, 100 \mathrm{lux}$ at cage level), at an ambient temperature (Ta) of $24^{\circ} \mathrm{C}$ with food and water ad libitum. The experimental protocol was approved by the Ethical Committee for Animal Research of the University of Bologna and by the Italian Ministry of Health, and was performed in accordance with the European Union (2010/63/UE) and the Italian Ministry of Health (January 27, 1992, No. 116) Directives, under the supervision of the Central Veterinary Service of the University of Bologna and the National Health Authority. The experimental protocol was assessed in order to minimize the number of animals used.

\section{Surgery}

Animals were anesthetized using an intramuscular (i.m.) injection of diazepam (Valium; F. HoffmannLa Roche 1td, Basel, Switzerland, $5 \mathrm{mg} / \mathrm{kg}$ ), followed by an intraperitoneal (i.p.) injection of ketamine- $\mathrm{HCl}$ (Ketavet; Parke-Davis, Detroit, MI, USA, 100 mg/kg). During surgery animals were implanted with: i) electrodes for electroencephalographic (EEG) and nuchal electromyographic (EMG) recording; ii) a femoral catheter for the telemetric recording of arterial pressure (AP) (PA-C40, DataSciences International, St.Paul, MN, USA) and heart rate determination (HR); iii) a thermistor (B10KA303N, Thermometrics Corporation, Northridge, CA, USA) mounted inside a stainless-steel needle (21 gauge) which was stereotaxically implanted above the left anterior hypothalamus for the recording of deep brain temperature $\left(\mathrm{T}_{\mathrm{b}}\right)$; iv) a microinjection guide cannula (C315G-SPC Plastics One Inc, Roanoke, VA, USA; internal cannula extension below guide: $+3.5 \mathrm{~mm}$ ), stereotaxically fixed in the right $\mathrm{LH}$ (according to Paxinos and Watson, 2007). After surgery, animals received both a subcutaneous injection of saline $(20 \mathrm{ml} / \mathrm{kg})$ and an i.m. injection of $1 \mathrm{ml} /$ $\mathrm{kg}$ of an antibiotic solution (penicillin G, $37500 \mathrm{IU}$; streptomycin-sulfate, $8750 \mathrm{IU}$ ).

After recovery from surgery, animals were housed in a Plexiglas cage which was placed within a thermoregulated and sound attenuated recording box, and were maintained at nlab for the whole experiment.

\section{Experimental Protocol}

All experiments were carried out on free-behaving rats and started at least seven days after their recovery from surgery. Animals were randomly assigned to two experimental groups ( $\mathrm{A}$ and $\mathrm{B}, \mathrm{n}=4$ for each group). During the experimental day, recordings 
started at L onset (09.00h). Following a two-hour recording session for the baseline, animals from group A were injected at $11.00 \mathrm{~h}$ with the selective $\mathrm{GABA}_{\mathrm{A}}$ receptor antagonist GABAzine (6-Imino3-(4-methoxyphenyl)-1(6H)-pyridazinebutanoic acid hydrobromide, $0.5 \mathrm{mM}, 100 \mathrm{nl}$ ), and at around $15.00 \mathrm{~h}$ (i.e. after the physiological parameters returned to baseline levels) with the cholinergic agonist Carbachol (Carbamoylcholine chloride, 10mM, 100nl), while animals from group B were only injected with $100 \mathrm{nl}$ of vehicle (Saline, SAL) at $11.00 \mathrm{~h}$.

Microinjections were performed with a Hamilton 5 $\mu \mathrm{l}$ gastight syringe (Hamilton Company, Bonaduz, Switzerland) connected to the internal cannula through one meter of microdialysis FEP tubing (ID0.12 mm OD $0.65 \mathrm{~mm}$, Microbiotech/se AB, Stockholm, Sweden) and placed in an infusion pump (MA 01746, Harvard Apparatus, Holliston, MA, USA; infusion rate $0.3 \mathrm{ml} / \mathrm{min}$ ). The cannula and the tubing were filled with either GBZ or CBL, both dissolved in vehicle solution (sterile-pyrogen free saline for parenteral injection (0.9\%), S.A.L.F. Bergamo, Italy), or with vehicle (SAL) solution only.

Ten minutes before the microinjection an operator gently inserted the internal cannula into the guide cannula. Animals were well trained to this procedure during the one-week adaptation period. After the correct positioning of the cannula, the microinjection was performed with the infusion pump (average duration: $30 \pm 5 \mathrm{~s}$ ).

At the end of each experimental session, animals were injected within the LH with $150 \mathrm{nl}$ of Fast Green 2\% dye. Animals were therefore anaesthetized according to the description in the surgery section and were transcardially perfused (4\% w/ vol paraformaldehyde). The brain was extracted, postfixed with $4 \%$ paraformaldehyde, cryoprotected ( $30 \% \mathrm{w} / \mathrm{vol}$ sucrose), and then sliced coronally on a cryostat $(60 \mu \mathrm{m})$. Sections containing the dye spot were digitally photographed, as shown in Figure 1. The analysis of the sections showed that all injections were properly carried out within the LH.

\section{Signals recording and analysis}

EEG, EMG and $\mathrm{T}_{\mathrm{b}}$ signals, recorded using insulated copper wires, were amplified (Grass 7P511L, AstroMed Inc, West Warwick (RI), USA), filtered (EEG: highpass $0.3 \mathrm{~Hz}$, lowpass $30 \mathrm{~Hz}$; EMG: highpass 100 $\mathrm{Hz}$, lowpass $1 \mathrm{KHz} ; \mathrm{T}_{\mathrm{b}}$ : highpass $0.5 \mathrm{~Hz}$ ), digitalized
(Micro MK 1401 II, CED, Cambridge, UK; acquisition rate: EEG: $1 \mathrm{KHz}$; EMG: $1 \mathrm{KHz}$; $\mathrm{b}$ : $100 \mathrm{~Hz}$ ), and acquired on a digital hard drive (Cerri et al., 2014).

The AP signal was telemetrically recorded, amplified and then stored on a hard drive (acquisition rate: $500 \mathrm{~Hz}$ ). HR was calculated on the basis of AP peak detection (Cerri et al., 2014). Wakefulness, NREM sleep, and REM sleep were scored visually by an operator (one-second resolution) according to standard criteria based on EEG, EMG, and Tb signals (Cerri et al., 2005b).

The effects of treatments on Wake-Sleep states (wakefulness, NREM sleep, and REM sleep), thermoregulatory $\left(\mathrm{T}_{\mathrm{b}}\right)$, and autonomic (MAP, HR) parameters were considered in the present study.

\section{Statistical analysis}

Statistical analysis was carried out using ANOVA (SPSS 21.0). A number of pre-planned non-orthogonal contrasts were made by means of the modified ttest $\left(t^{*}\right)$, where the alpha level was adjusted by the "sequential" Bonferroni correction (Holm et al., 1979).

For each variable, a separate analysis was carried out for data taken as absolute values (pre-injection baseline and post-injection levels) and for data taken as the difference between post-injection and preinjection baseline levels.

In the first case (absolute values), results were analyzed using one-way ANOVA with repeated measures. Analysis was carried out separately for each variable and for each injected substrate in order to study the effects of each treatment on the different variables. Baseline levels were assessed in the 30-min preceding the injection (injection time was taken as 0 ) for the Wake-Sleep states and in the 10-min time period which preceded the injection for thermoregulatory and autonomic parameters. The factor "time" was considered as a within-group factor, with five levels for the Wake-Sleep states (-30-0 min, baseline; 0-30 $\mathrm{min}$; 30-60 $\mathrm{min}$; 60-90 $\mathrm{min}$; 90-120 min) and four levels for thermoregulatory and autonomic parameters (-10-0 min, baseline; 0-30 min; 30-60 $\mathrm{min}$; 60-90 $\mathrm{min}$ ). Four non-orthogonal contrasts were carried out for the analysis of the effects on the Wake-Sleep states, and three for the effects on thermoregulatory and autonomic parameters (the value of each 30-min post-injection time interval was compared with the pre-injection baseline). 


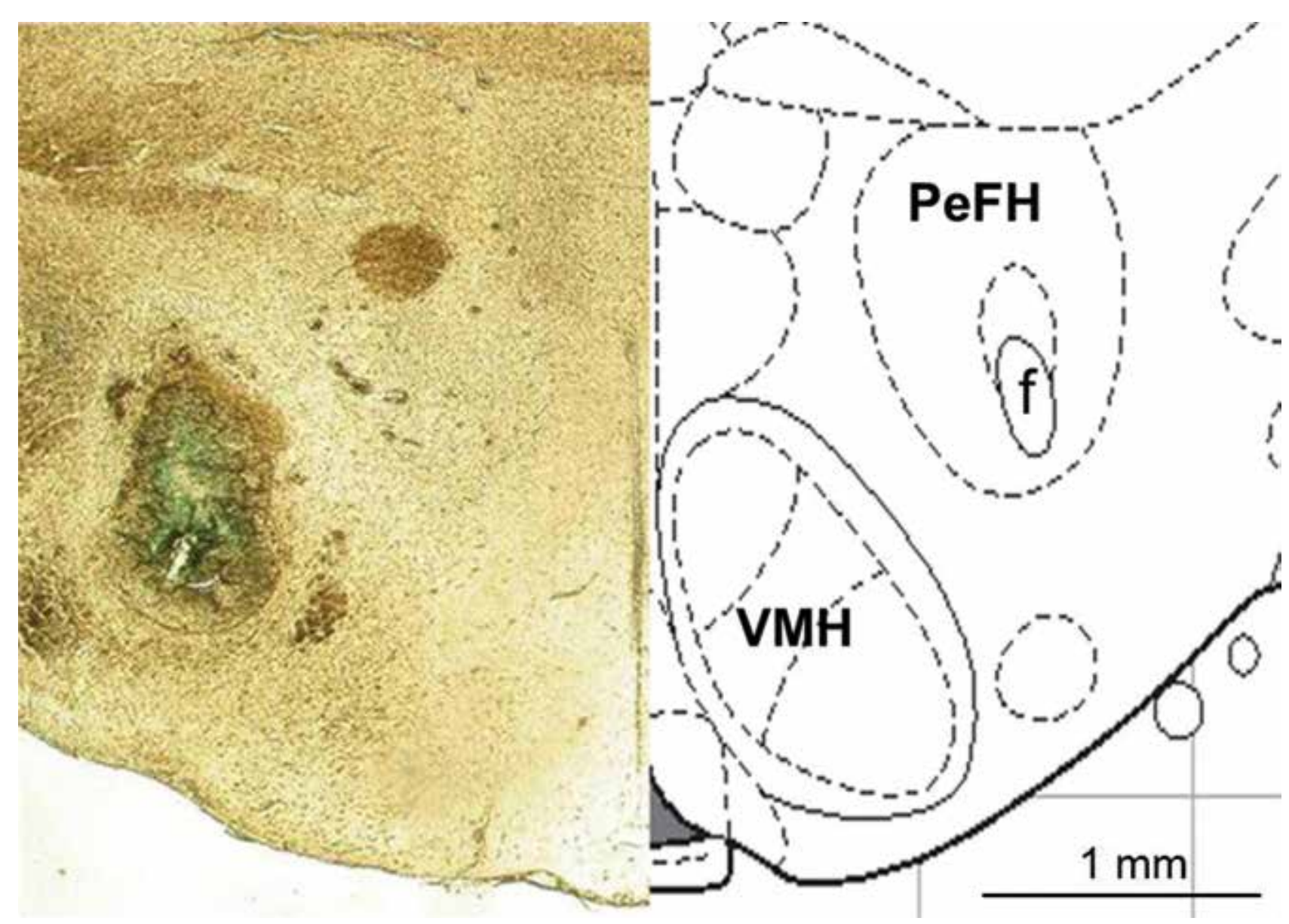

Fig. 1. - On the left, sample of the injection site location in the lateral hypothalamus of one animal. On the right, schematic drawing of the injection area (plate from Paxinos and Watson, 2007). f: fornix; PeFH: peri-fornical hypothalamic area; VMH: ventro-medial hypothalamus.

In the second case (differential values between the levels in each of the 30-min post-injection time intervals and the pre-injection baseline), results were analyzed using two-way ANOVA with repeated measures on one factor. The analysis was carried out separately for each variable in order to compare the effects of the different treatments. The factors were considered as follows: i) factor "time", considered for repeated measures, with four levels for Wake-Sleep states (0-30 min; 30-60 min; 60-90 min; 90-120 min) and three levels for thermoregulatory and autonomic parameters (0-30 min; 30-60 min; 60-90 min); ii) factor "treatment" with three levels: SAL, GBZ and CBL. Eight non-orthogonal contrasts were carried out for Wake-Sleep states and six were made for thermoregulatory and autonomic parameters (for each 30-min post-injection interval, values following the administration of either GBZ or CBL injection were compared to those following SAL).
Throughout all analyses, differences were considered to be statistically significant when $\mathrm{P}<0,05$.

\section{Results}

\section{Wake-Sleep effects}

As shown in Figure 2A, GBZ microinjection within the LH significantly increased the time spent in wakefulness in the 0-30 $\mathrm{min}\left(29.4 \pm 0.3 \mathrm{~min}, \mathrm{t}_{(12)}=-4.94\right)$, $30-60 \mathrm{~min}\left(29.4 \pm 0.2 \mathrm{~min}, \mathrm{t}^{*}{ }_{(12)}=-4.96\right)$, and $60-90$ $\min \left(25.0 \pm 3.5 \mathrm{~min}, \mathrm{t}^{*}{ }_{(12)}=-3.56\right)$ post-injection time intervals when compared to the pre-injection baseline levels $(13.2 \pm 2.4 \mathrm{~min})$. On the contrary, sleep occurrence was almost suppressed in the first hour following injection. In particular, the time spent in NREM sleep (Figure 2C) was significantly lower in the 0-30 $\min \left(0.3 \pm 0.3 \mathrm{~min}, \mathrm{t}^{*}{ }_{(12)}=4.82\right), 30-60 \min (0.2 \pm 0.2$ 
$\left.\min , \mathrm{t}_{(12)}=4.84\right)$ and $60-90 \mathrm{~min}\left(4.5 \pm 3.4 \mathrm{~min}, \mathrm{t}_{(12)}\right.$ $=3.32$ ) post-injection time-intervals compared to the pre-injection baseline period (14.4 $\pm 2.2 \mathrm{~min})$. REM sleep was suppressed for one hour after GBZ administration (Figure 2E), although, paradoxically, this effect didn't reach statistical significance. The time spent in wakefulness, NREM sleep, and REM sleep returned to around baseline levels in the 90-120 min post-injection time interval.

The administration of CBL within the LH also significantly increased the time spent in wakefulness (Figure $2 \mathrm{~A})$ in the $0-30 \mathrm{~min}\left(18.6 \pm 5.5 \mathrm{~min}, \mathrm{t}^{*}{ }_{(12)}=\right.$ -3.71) post-injection time interval compared to the pre-injection baseline $(6.5 \pm 2.1 \mathrm{~min})$, and reduced the time spent in NREM sleep (Figure 2C) in the first 30-min post-injection time interval $(6.3 \pm 2.3$ min, $\left.t^{*}{ }_{(12)}=3.07\right)$ compared to the pre-injection baseline $(20.1 \pm 2.2 \mathrm{~min})$. The administration of CBL, similarly to that of GBZ, led to a REM sleep suppression (Figure 2E), which was limited, however, to the first 30-min post-injection interval.

Following SAL injection, an increase in the time spent in wakefulness and a decrease in the time spent in both NREM sleep and REM sleep were observed during the post-injection period (Figure 2A, 2C, and $2 \mathrm{E}$, respectively). However, these changes were not statistically significant for any of the 30-min postinjection intervals when compared to the respective pre-injection baseline. Interestingly, as shown in Figure 2B, 2D, and $2 \mathrm{~F}$ (in which the differentials for each of the four 30-min post-injection time periods with the pre-injection baseline levels are considered), no statistically significant differences were observed between the effects induced by the injection of either CBZ or CBL and those induced by SAL in any of the three Wake-Sleep states.

\section{Thermoregulatory and autonomic effects}

As shown in Figure 3A, following GBZ administration a significant increase in $T_{b}$ was observed in the $0-30 \mathrm{~min}\left(38.47 \pm 0.25^{\circ} \mathrm{C}, \mathrm{t}^{*}{ }_{(9)}=-6.53\right), 30-60$ $\min \left(38.50 \pm 0.29^{\circ} \mathrm{C}, \mathrm{t}^{*}{ }_{(9)}=-6.70\right)$, and $60-90 \mathrm{~min}$ $\left(38.00 \pm 0.33^{\circ} \mathrm{C}, \mathrm{t}^{*}{ }_{(9)}=-3.55\right)$ post-injection time intervals compared to the 10-min pre-injection baseline $\left(37.44 \pm 0.34^{\circ} \mathrm{C}\right)$. HR levels (Figure $3 \mathrm{C}$ ) were also significantly higher in the first hour interval which followed the injection (0-30 min: 466.5 \pm 25.0 bpm, $\mathrm{t}_{(9)}^{*}=-5.06$; 30-60 min: $422.8 \pm 19.0 \mathrm{bpm}, \mathrm{t}_{(9)}$ $=-3.06)$ compared to the pre-injection baseline
(356.1 $\pm 15.6 \mathrm{bpm})$. An apparent increase, although not statistically significant, was also observed in MAP levels (Figure 3E) in the 0-30 min postinjection time interval compared to the pre-injection baseline.

Overall, similar effects were induced by the microinjection of CBL. In fact, $\mathrm{T}_{\mathrm{b}}$ (Figure $3 \mathrm{~A}$ ) increased in the $0-30 \mathrm{~min}\left(38.04 \pm 0.03^{\circ} \mathrm{C}, \mathrm{t}^{*}{ }_{(9)}=-6.35\right)$, the $30-60$ $\min \left(37.76 \pm 0.20^{\circ} \mathrm{C}, \mathrm{t}^{*}{ }_{(9)}=-4.65\right)$, and the $60-90 \mathrm{~min}$ $\left(37.76 \pm 0.20^{\circ} \mathrm{C}, \mathrm{t}^{*}{ }_{(9)}=-4.64\right)$ post-injection time intervals, while HR (Figure 3C) and MAP (Figure 3E) were increased in the $0-30 \mathrm{~min}$ post-injection time interval (HR: 389.9 $\pm 25.6 \mathrm{bpm}, \mathrm{t}^{*}{ }_{(9)}=-5.67$; MAP: $\left.106.1 \pm 3.3 \mathrm{mmHg}, \mathrm{t}_{(9)}=-9.09\right)$ compared to the respective pre-injection baseline $\left(\mathrm{T}_{\mathrm{b}}: 36.98 \pm 0.03^{\circ} \mathrm{C}\right.$; HR: $309.6 \pm 14.3 \mathrm{bpm}$; MAP: $92.2 \pm 4.4 \mathrm{mmHg}$ ). No statistically significant effects were induced by SAL microinjection on either $T_{b}$, HR or MAP levels (Figure 3A, 3C, and 3E, respectively).

Differently from what was observed for the WakeSleep states, the statistical analysis of data calculated as the differential from the pre-injection baseline levels for each of the three 30-min post-injection time periods showed the presence of significant differences between the effects induced by either GBZ or CBL and those induced by SAL administration. As shown in Figure 3B, 3D, and 3F, in the first hour which followed GBZ injection, the variation from the respective pre-injection baseline was significantly different for both $\mathrm{T}_{\mathrm{b}}, \mathrm{HR}$, and MAP compared to SAL. In fact, the thermoregulatory and autonomic parameters significantly increased following GBZ in both the $0-30 \mathrm{~min}\left(\mathrm{~T}_{\mathrm{b}}: 1.03 \pm 0.20^{\circ} \mathrm{C}, \mathrm{t}^{*}{ }_{(18)}\right.$ = 7.78; HR: $110.4 \pm 30.7 \mathrm{bpm}, \mathrm{t}_{(18)}=7.87$; MAP: $\left.21.1 \pm 4.7 \mathrm{mmHg}, \mathrm{t}^{*}{ }_{(18)}=6.68\right)$ and the $30-60 \mathrm{~min}\left(\mathrm{~T}_{\mathrm{b}}\right.$ : $1,06 \pm 0.20^{\circ} \mathrm{C}, \mathrm{t}^{*}{ }_{(18)}=7.13$; HR: $66.7 \pm 26.7 \mathrm{bpm}, \mathrm{t}^{*}{ }_{(18)}$ $=4.98$; MAP: $\left.12.0 \pm 5.1 \mathrm{mmHg}, \mathrm{t}_{(18)}=4.21\right)$ postinjection time intervals compared to SAL (0-30 min: $\mathrm{T}_{\mathrm{b}}: 0.19 \pm 0.22^{\circ} \mathrm{C}$; HR: $21.73 \pm 16.93 \mathrm{bmp}$; MAP: $3.71 \pm 3.61 \mathrm{mmHg}$. 30-60 min: $\mathrm{T}_{\mathrm{b}}: 0.29 \pm 0.25^{\circ} \mathrm{C}$; HR: $10.60 \pm 12.45 \mathrm{bmp}$; MAP: $1.02 \pm 1.73 \mathrm{mmHg}$ ). A significantly larger effect than that observed following SAL injection was also observed following CBL injection concerning $\mathrm{T}_{\mathrm{b}}$ levels, in the $0-30 \mathrm{~min}$ $\left(1.06^{\circ} \pm 0.21 \mathrm{t}^{*}{ }_{(18)}=8.06\right)$ and in the $30-60 \mathrm{~min}$ $\left(0.77^{\circ} \pm 0.23 \mathrm{t}_{(18)}=4.50\right)$ post-injection time intervals, and both HR and MAP levels in the 0-30min post-injection time interval (HR: $80.3 \pm 22.9 \mathrm{bpm}$ $\mathrm{t}^{*}{ }_{(18)}=5.19$, MAP: $\left.13.9 \pm 1.4 \mathrm{mmHg} \mathrm{t}_{(18)}^{*}=3.89\right)$. 

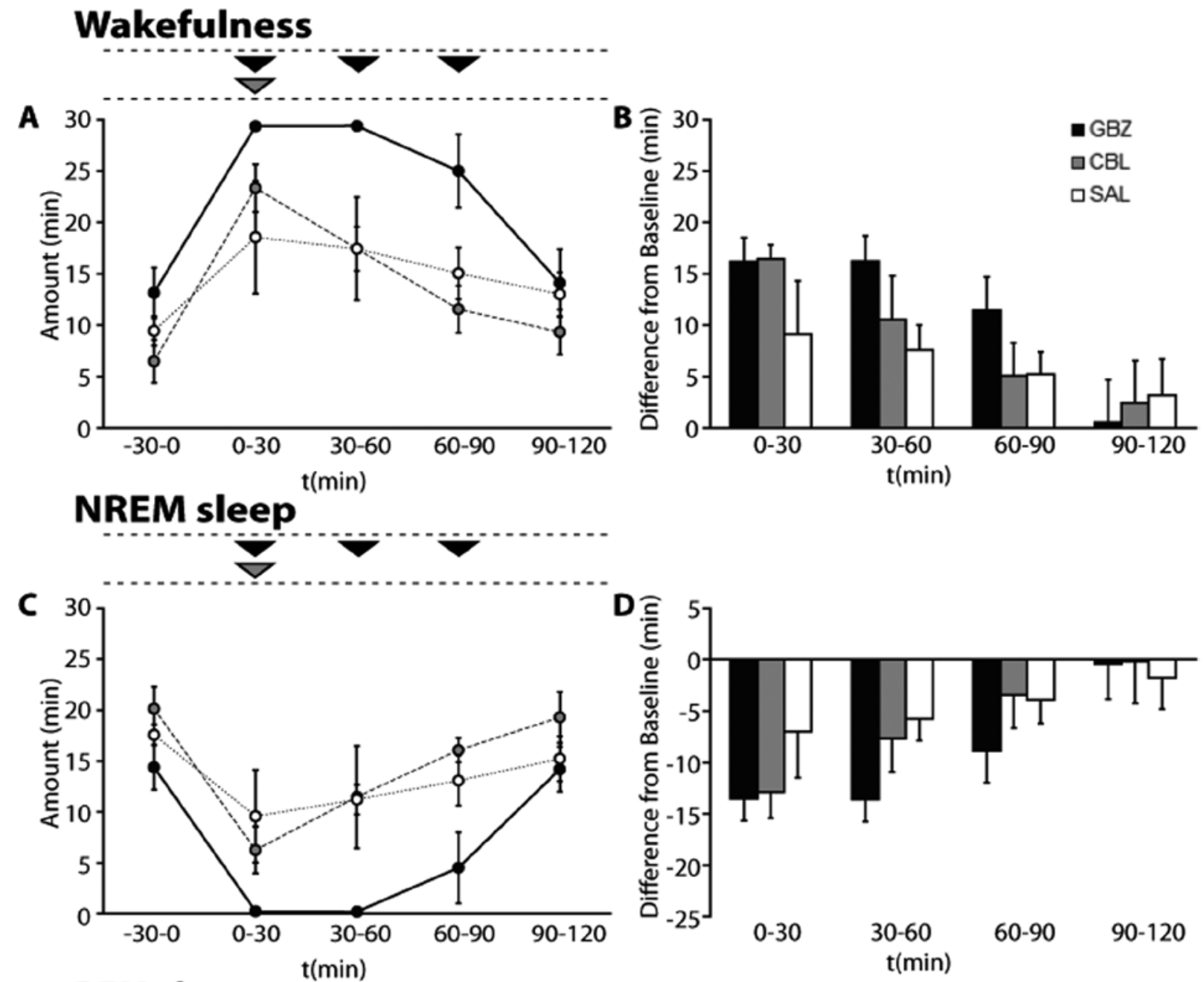

REM sleep
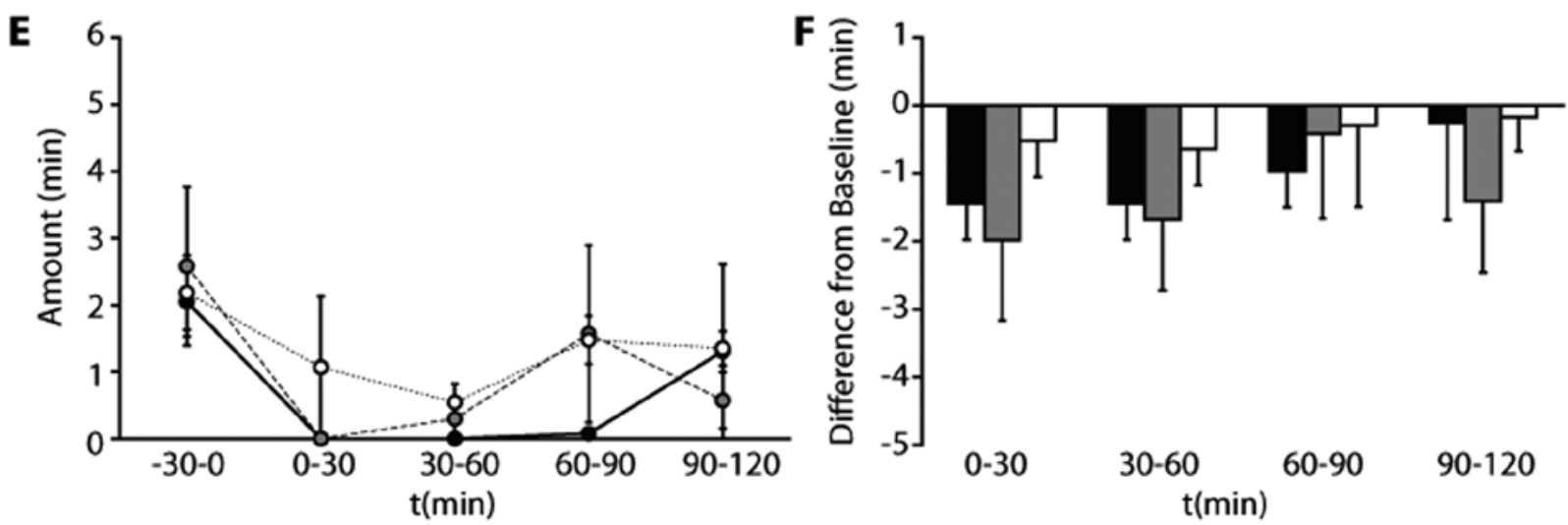

Fig. 2. - Wake-Sleep effects of the unilateral microinjection in the lateral hypothalamus of the rat, of either the $G A B A_{A}$ antagonist GABAzine (GBZ, $1 \mathrm{mM}, 100 \mathrm{nl}$, black), the cholinergic agonist Charbacol (CBL, 1mM, 100nl, grey), or Saline (SAL, 100nl, white). In the left column, the time spent in either wakefulness (A), NREM sleep (C), or REM sleep (E) is shown (amount in $\mathrm{min}$ ) for the 30-min interval pre-injection baseline period ( $-30-0$ min, time of injection taken as 0 ) and for four subsequent 30-min interval post-injection periods (0-30 min; 30-60 min; 60-90 min; $90-120$ min). In the right column, the differential value between the time spent in each Wake-Sleep state in each of the four 30 -min interval post-injection periods and the respective pre-injection baseline is shown for either wakefulness (B), NREM sleep (D), or REM sleep (F). All data are expressed as mean \pm S.E.M. In the left column, the triangle between the dashed lines indicates a significant difference $(p<0.05)$ between a 30-min post-injection period and the respective pre-injection baseline, following either GBZ (black) or CBL (grey). 


\section{Discussion}

To the best of our knowledge, this pilot study is the first showing that the activation of cholinergic receptors within the LH evokes significant autonomic and behavioral responses in the free-behaving rat. In view of our modest sample size we are aware that the current results are in clear need of replication.

In the present study, the effects of the cholinergic agonist CBL were compared to those induced by the $\mathrm{GABA}_{\mathrm{A}}$ antagonist GBZ, the latter being expected to be larger since GBZ involves a wider population of LH neurons. The results confirmed this view. Also, the activating effects of both drugs showed a maximum, which was apparently larger for GBZ compared to CBL in the 30 min following their LH administration, and a parallel decline afterwards. Although this outcome may be easily explained by a difference in the size of the population of neurons involved, it should be kept in mind that our experimental approach did not address the issue of different pharmacokinetics for the drugs employed in the study.

The distribution of the different behavioral states following CBL and GBZ administration developed in time with the pattern described above, characterized by larger effects of GBZ compared to CBL and, on the whole, by an increase in wakefulness and a decrease in sleep. Unexpectedly, milder but similar effects were induced by the administration of the drug vehicle (SAL). In spite of this, the effects of SAL administration were sufficient to mask any statistical significance in the comparisons concerning the effects induced by CBL and GBZ. A normal saline solution is considered a standard vehicle for local microinjections, but we have previously noted a modest activating effect of SAL on LH neurons (Cerri et al., 2014). Some experimental results show that ORX neurons are particularly sensitive to changes in extracellular $\mathrm{pH}$, doubling their firing rate for a 0.1 unit increase (Williams et al., 2007). The effects of normal saline may very likely arise from the fact that the solution, that does not contain a buffer, may locally dilute the extracellular bicarbonate and cause, in an environment where $\mathrm{CO}_{2}$ is constant, a decrease in $\mathrm{pH}$ that is sufficient to increase the activity of the surrounding sensitive neurons (Reddi, 2013).

Although ORX neurons constitute the arousal system of LH (Lin et al., 2011), these data suggest that their putative activation within the subset of the CBL-sensitive neurons is not sufficient to promote a period of wakefulness comparable to that evoked by GABAzine. Thus, either the cholinergic inputs from BF described by Sakurai et al. (2005) are not sufficient to activate the whole set of wake-promoting ORX neurons or, as suggested by Henny and Jones (2006), the cholinergic afferents to LH do not mainly originate from $\mathrm{BF}$.

A detailed analysis of the effects of CBL and GBZ on Sleep show that the stronger arousal elicited by GBZ suppressed both NREM sleep and REM sleep, whilst the effects of CBL on NREM sleep were milder in comparison to those on REM sleep. The sensitivity of REM sleep mechanisms to an arousal elicited by LH activation may be explained by the well-known inhibitory effect that the activation of thermogenesis elicits on the occurrence of REM sleep (Parmeggiani, 2003; Cerri et al., 2005; Amici et al., 2013). It may be noted that the significant increase of brain temperature induced by both CBL and GBZ is fundamentally coincident with the time course of REM sleep inhibition.

As far as autonomic effects are concerned, the results showed a large increase in both brain temperature and HR and a more modest variation in AP. The size and coherence of temperature and HR variations point to the role played by LH in thermoregulation (Cerri and Morrison, 2005; Cerri et al., 2014), suggesting that the activation of the subset of CBL-sensitive neurons is sufficient to activate an autonomic outflow that is able to fully sustain thermogenesis. In fact, it has been shown that the disinhibition of LH neurons in the anaesthetized rat induces a large increase in HR that is mediated by neurons in the Raphe Pallidus (RPa) (Cerri and Morrison, 2005). Thus, the subset of CBL-sensitive neurons of LH may also project to neurons within the RPa, a key relay area in the central circuit that controls thermogenesis (Cerri et al., 2010; Morrison et al., 2014). The finding that ORX neurons projecting to pre-sympathetic motor neurons in $\mathrm{RPa}$ activate brown fat thermogenesis (Tupone et al., 2011) is in favor of the presence of ORX neurons within the CBL-sensitive cells of LH.

Arterial blood pressure is mainly controlled by the sympathetic premotor neurons located within the Rostral Ventrolateral Medulla (RVLM) (Dampney et al., 1984). This area receives inhibitory inputs 

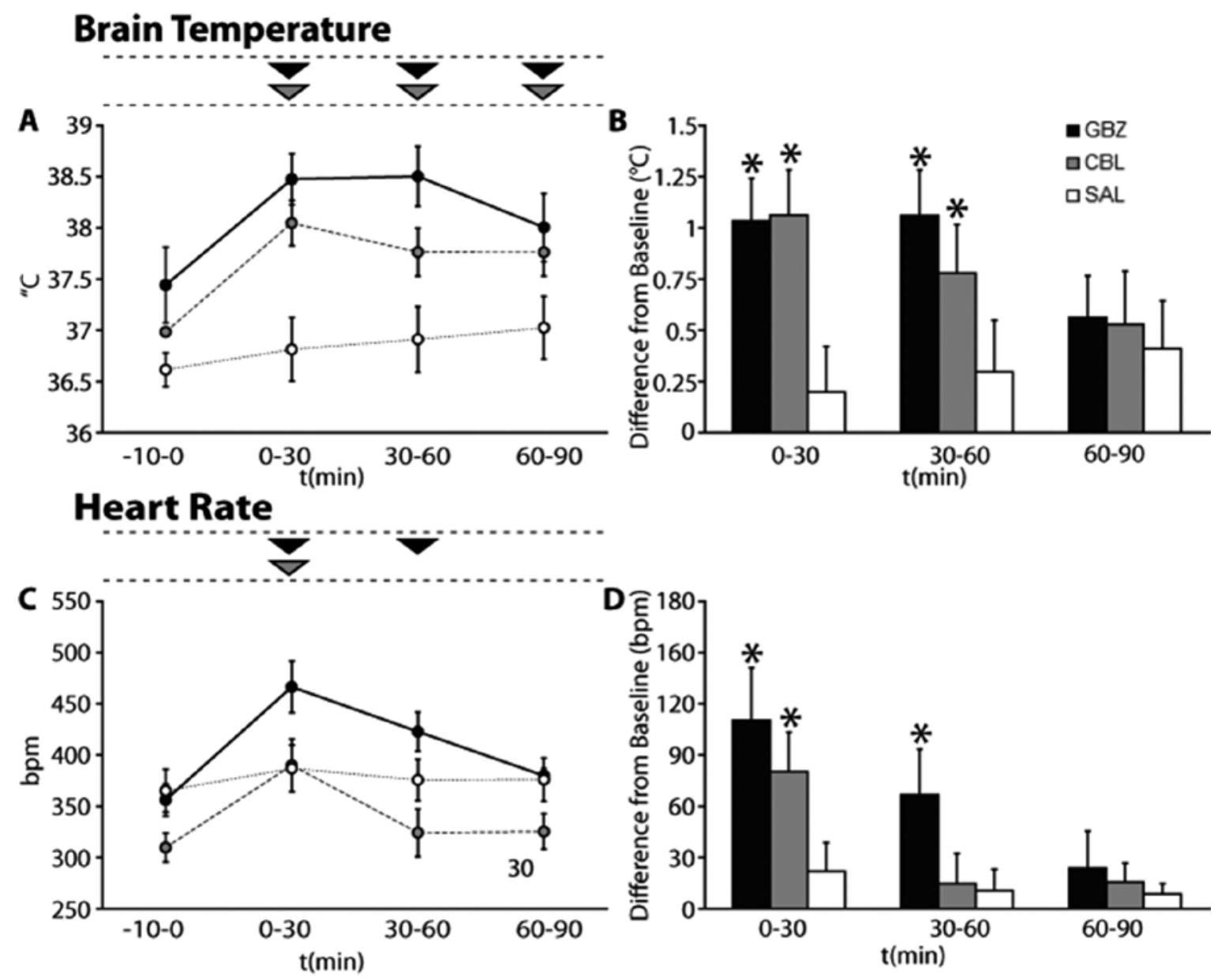

\section{Mean Arterial Pressure}
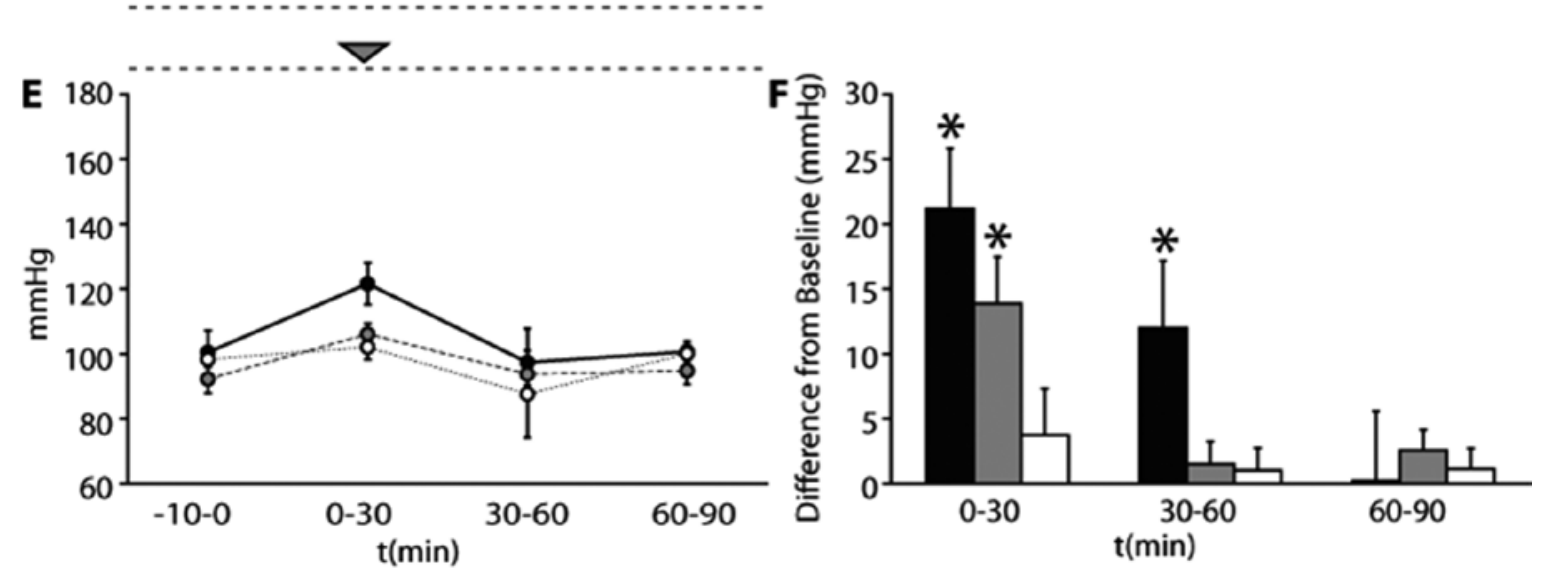

Fig. 3. - Thermoregulatory and autonomic effects of the unilateral microinjection in the lateral hypothalamus of the rat of either the $\mathrm{GABA}_{\mathrm{A}}$ antagonist GABAzine $(\mathrm{GBZ}, 1 \mathrm{mM}, 100 \mathrm{nl}$, black), the cholinergic agonist Charbacol (CBL, $1 \mathrm{mM}, 100 \mathrm{nl}$, grey), or Saline (SAL, 100nl, white). In the left column, the changes in deep brain temperature ( $T_{b}$, ${ }^{\circ} \mathrm{C}$; A), heart rate (HR, bpm; C), or mean arterial pressure (MAP, mmHg; E) are shown, for the 10-min interval preinjection baseline period (-10-0 min, time of injection taken as 0 ) and four subsequent 30-min interval post-injection periods (0-30 min; 30-60 $\mathrm{min}$; 60-90 min; 90-120 min). In the right column, the differential value (mean \pm S.E.M.) between the level of each of the three thermoregulatory and autonomic variables in each of the four 30-min interval post-injection periods and the respective pre-injection baseline is shown for either $T_{b}(B), H R(D)$, or MAP (F). All data are expressed as mean \pm S.E.M. In the left column, the triangle between the dashed lines indicates a significant difference $(p<0.05)$ between a 30-min post-injection period and the respective pre-injection baseline, following either GBZ (black) or CBL (grey). In the right column, * indicates a significant difference $(p<0.05)$ between the effects induced by either GBZ or CBL compared to those induced by SAL. 
from LH (Bowman et al., 2013), which may explain why the increase observed in AP, although modest, does not appear to be compensated by a baroreflex activation. Within this frame, the rise of AP values following the administration of the two drugs may be explained as the consequence of an increment in cardiac output induced by the large increase in HR. In conclusion, the results of this work show that, in the free-behaving rat, the activation of LH can elicit an arousal that is characterized by behavioral and autonomic responses which appear to be mainly coordinated by cold-defense mechanisms. This kind of integrative activity may be elicited at a lower intensity by cholinoceptive cells, some of which are ORX neurons.

\section{Acknowledgements}

This work has been supported by the Ministero dell'Istruzione, dell'Università e della Ricerca (MIUR)- Italy, and by the Alma Mater Studiorum - University of Bologna. The authors wish to thank Ms Melissa Stott for reviewing the English text.

\section{References}

Alexandre C., Andermann M.L., Scammell T.E. Control of arousal by the orexin neurons. Curr. Opin. Neurobiol., 23: 752-759, 2013.

Amici R., Cerri M. and Parmeggiani P.L. Overview of Physiological Processes During Sleep. pp. 385389. In: Kushida C.A. (Eds.) The Encyclopedia of Sleep. Waltham MA, Academic Press, 2013.

Berthoud H.R. and Munzberg H. The lateral hypothalamus as integrator of metabolic and environmental needs: from electrical self-stimulation to opto-genetics. Physiol. Behav., 104: 29-39, 2011.

Bowman B.R., Kumar N.N., Hassan S.F., McMullan S., Goodchild A.K. Brain sources of inhibitory input to the rat rostral ventrolateral medulla. J. Comp. Neurol., 521: 213-232, 2013.

Cerri M. and Morrison S.F. Activation of lateral hypothalamic neurons stimulates brown adipose tissue thermogenesis. Neuroscience, 135: 627-638, 2005.

Cerri M., Ocampo-Garces A., Amici R., Baracchi F., Capitani P., Jones C.A., Luppi M., Perez E., Parmeggiani P.L., Zamboni G. Cold exposure and sleep in the rat: effects on sleep architecture and the electroencephalogram. Sleep, 28: 694-705, 2005.
Cerri M., Zamboni G., Tupone D., Dentico D., Luppi M., Martelli D., Perez E., Amici R. Cutaneous vasodilation elicited by disinhibition of the caudal portion of the rostral ventromedial medulla of the free-behaving rat. Neuroscience, 165: 984-95, 2010.

Cerri M., Del Vecchio F., Mastrotto M., Luppi M., Martelli D., Perez E., Tupone D., Zamboni G., Amici R. Enhanced slow-wave EEG activity and thermoregulatory impairment following the inhibition of the lateral hypothalamus in the rat. PLoS One, 9: e112849, 2014.

Clement O., Sapin E., Libourel P.A., Arthaud S., Brischoux F., Fort P., Luppi P.H. The lateral hypothalamic area controls paradoxical (REM) sleep by means of descending projections to brainstem GABAergic neurons. J. Neurosci., 32: 16763-16774, 2012.

Dampney R.A., Goodchild A.K., Tan E. Identification of cardiovascular cell groups in the brain stem. Clin. Exp. Hypertens. A., 6: 205-220, 1984.

Eggermann E., Serafin M., Bayer L., Machard D., Saint-Mleux B., Jones B.E., Mühlethaler M. Orexins/hypocretins excite basal forebrain cholinergic neurones. Neuroscience, 108:177-81, 2001.

Henny P., and Jones B.E. Innervation of orexin/ hypocretin neurons by GABAergic, glutamatergic or cholinergic basal forebrain terminals evidenced by immunostaining for presynaptic vesicular transporter and postsynaptic scaffolding proteins. $J$. Comp. Neurol., 499: 645-61, 2006.

Holm S. A simple sequentially rejective multiple test procedure. Scand. J. Stat., 6: 65-70, 1979.

Lee M.G., Hassani O.K., Alonso A., Jones B.E. Cholinergic basal forebrain neurons burst with theta during waking and paradoxical sleep. $J$. Neurosci., 25: 4365-9, 2005.

Li F.W., Deurveilher S., Semba K. Behavioural and neuronal activation after microinjections of AMPA and NMDA into the perifornical lateral hypothalamus in rats. Behav. Brain. Res., 224: 376-386, 2011.

Lin J.S., Anaclet C., Sergeeva O.A., Haas H.L. The waking brain: an update. Cell. Mol. Life Sci., 68: 2499-2512, 2011.

Morrison S.F., Madden C.J., Tupone D. Central neural regulation of brown adipose tissue thermogenesis and energy expenditure. Cell. Metab., 19: 741-756, 2014.

Parmeggiani P.L. Thermoregulation and sleep. Front. Biosci., 8: s557-567, 2003.

Paxinos G. and Watson C. The rat brain in stereotaxic coordinates 6th Edition. 2007, San Diego: Elsevier. 
Reddi B.A. Why is saline so acidic (and does it really matter?). Int. J. Med. Sci., 10: 747-750, 2013.

Shirasaka T., Kunitake T., Takasaki M., Kannan H. Neuronal effects of orexins: relevant to sympathetic and cardiovascular functions. Regul. Pept., 104: 91-95, 2002.

Sakurai T., Nagata R., Yamanaka A., Kawamura H., Tsujino N., Muraki Y., Kageyama H., Kunita S., Takahashi S., Goto K., Koyama Y., Shioda S., Yanagisawa M. Input of orexin/hypocretin neurons revealed by a genetically encoded tracer in mice. Neuron, 46: 297-308, 2005.
Tupone D., Madden C.J., Cano G., Morrison S.F. An orexinergic projection from perifornical hypothalamus to raphe pallidus increases rat brown adipose tissue thermogenesis. J. Neurosci., 31: 15944-15955, 2011.

Yoshida K., McCormack S., España R.A., Crocker A., Scammell T.E. Afferents to the orexin neurons of the rat brain. J. Comp. Neurol., 494: 845-61, 2006.

Williams R.H., Jensen L.T., Verkhratsky A., Fugger L., Burdakov D. Control of hypothalamic orexin neurons by acid and CO2. Proc. Natl. Acad. Sci.U.S.A., 104: 10685-10690, 2007. 